\title{
Zur Psychidenfauna Albaniens unter Berücksichtigung der Albanien- Expedition 1961 des Deutschen Entomologischen Institutes (Lepidoptera: Psychidae)
}

\author{
Mit 3 Figuren und 1 Karte
}

Michael WeidLICH ${ }^{1}$

${ }^{1}$ Lindenallee 11, 15898 Neißemünde OT Ratzdorf, Deutschland. - dr.michael.weidlich@gmx.de Published on 2013-12-20

\section{Zusammenfassung}

In der vorliegenden Arbeit wird eine Übersicht zur Psychidenfauna Albaniens gegeben und die entsprechende Literatur ausgewertet. Die ersten Nachweise stammen aus den frühen Jahren des 20. Jahrhunderts. Bereits 1931 wurde eine zusammenfassende faunistische Arbeit veröffentlicht. Die Albanienexpedition des Deutschen Entomologischen Institutes in Eberswalde im Jahre 1961 erbrachte weitere Nachweise. Ab 2006 war der Verfasser mehrmals zu Psychidenstudien in Albanien.

Gegenwärtig liegen Nachweise von Psychiden für insgesamt 77 Fundorte aus 24 Provinzen vor. Bis zum Jahre 1931 und durch die Albanienexpedition 1961 waren insgesamt 16 Arten bekannt geworden. Der Verfasser konnte im Beobachtungszeitraum fünf weitere Psychidenarten im Land entdecken. Neben der Erstbeschreibung von Epichnopterix hellenidensis sind dies Pseudobankesia sp., Luffia lapidella (Goeze, 1783), Stichobasis helicinoides (HeylaERTs, 1879) und Oiketicoides lutea (Staudinger, 1871). Bis zum heutigen Zeitpunkt sind somit 21 Psychidenarten nachgewiesen worden. Taleporia politella (OCHSEnHeImer, 1816) und Acanthopsyche zelleri (MANn, 1855) müssen aus zoogeographischen Gesichtspunkten heraus als Faunenbestandteil gestrichen werden, weitere Taxa werden diskutiert. Die bisherigen Literaturangaben für Albanien von Proutia betulina (ZELler, 1839), Psyche casta (PALLAs, 1767) und Sterrhopterix fusca (HAworTH, 1809) müssen revidiert werden, da sie von Fundorten stammen, die jetzt zu den Nachbarländern Kosovo und Mazedonien gehören. Ergänzend wird bei allen albanischen Arten eine Übersicht zur ihrer Verbreitung in den Nachbarländern gegeben.

\section{Summary}

In the present paper an overview of the albanian Psychid-fauna is given and evaluated the relevant literature. The first records date from the early years of the 20th Century and a summary of the species inventory was given in 1931. The expedition of the German Entomological Institute in Eberswalde to Albania in 1961 provided further evidence. Since 2006, the author was several times in Albania for studies of Psychid-fauna.

The records of Psychids are currently a total of 77 localities in 24 provinces spread over the entire country. Until 1931 and supplemented by the Albania Expedition 1961 a total of 16 species were known. The author found for the first time five species for the albanian faune. In addition to the initial description of Epichnopterix hellenidensis are Pseudobankesia sp., Luffia lapidella (Goeze, 1783), Stichobasis helicinoides (Heylaerts, 1879) and Oiketicoides lutea (Staudinger, 1871). Until today 21 Psychid-species have been detected. Taleporia politella (Ochsenheimer, 1816) and Acanthopsyche zelleri (MANN, 1855) must be delete from the faunistical list from zoogeographical aspect, other taxa are discussed. The current literature of Albania for Proutia betulina (ZELler, 1839), Psyche casta (PALlas, 
1767) and Sterrhopterix fusca (HAworTh, 1809) need to be revised, since they come from sites that are currently among the neighboring Kosovo and Macedonia. In addition, an overview of all Albanian species to occuring in the neighboring countries is given.

\section{Key words}

Albania, faunistic review, Psychidae, Lepidoptera

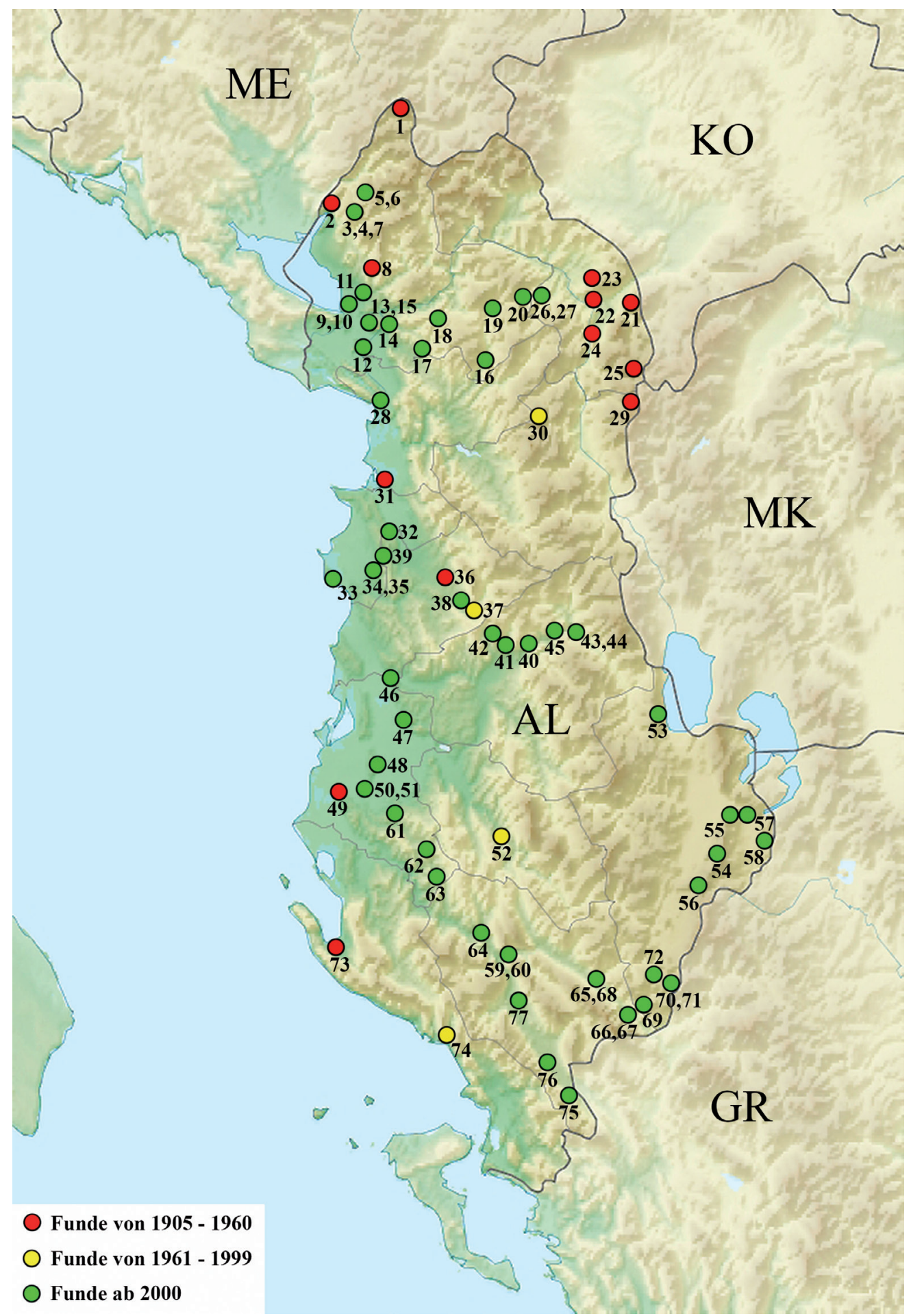

Karte 1: Lage der albanischen Psychidenfundorte mit Beobachtungszeiträumen (verändert nach http://en.wikipedia.org/wiki/ File:Albania_relief_location_map.jpg) Rote Punkte = Funde von 1905 - 1960; gelbe Punkte = Funde von 1961 - 1999; grüne Punkte $=$ Funde $\mathrm{ab} 2000$. 


\section{Einleitung}

Die Erforschung der Schmetterlingsfauna Albaniens begann erst relativ spät und kann auf den Beginn des 19. Jahrhunderts datiert werden. Die ersten Nachweise zur Psychidenfauna Albaniens gehen wohl auf STURAnY aus dem Jahre 1905 zurück (REBEL, 1914b: 37; REBEL \& ZERNY, 1931: 44, 123, 333).

Verschiedene Einzelnachweise finden sich dann in der Literatur und auch die Albanien-Expedition 1961 des Deutschen Entomologischen Institutes in Eberswalde, nachfolgend DEI genannt, steuerte einen Beitrag zur Erforschung der Psychidenfauna des Landes bei. Einzelheiten zur Expedition finden sich bei Friese \& KöNIGSMANN (1962). Dazu ist bereits ein Teil der Ausbeute mit den Arten Diplodoma laichartingella (Goeze, 1783) und Eumasia parietariella (HeydenreICH, 1851) („Micropsychina") publiziert worden (PETERSEN, 1964: 378-379). Die weiteren Psychiden der Expedition wurden bisher nicht bearbeitet und bilden jetzt einen Gegenstand der Veröffentlichung. Offenbar sind dann über vier Dezennien hinweg keine neuen Psychidenfunde bekannt geworden. Auch eine diesbezügliche Anfrage an P. HäTTENSCHWILER (Uster/Schweiz) konnte diese Annahme bestätigten (in litt. HÄTTENSCHWILER, 2013).

In den Jahren 2006, 2008, 2010, 2011, 2012 und 2013 weilte der Autor jeweils für einige Tage im Frühjahr im Lande.

Trotz alledem sind die Kenntnisse über die Psychidenfauna bis zum heutigen Tage als lückenhaft einzuschätzen. Insbesondere in den Hochgebirgslagen sind durchaus noch weitere interessante Arten zu erwarten.

\section{Die Fundorte albanischer Psychiden (= Fundortverzeichnis)}

Die administrative Gliederung von Albanien umfasst 12 Länder bzw. Grafschaften (Qarku). Diese untergliedern sich jeweils in verschiedene Provinzen bzw. Distrikts (Rrethe); ab 1991 bestehen 36 Provinzen. Die Aussengrenzen Albaniens unterlagen in der Vergangenheit vielfachen Veränderungen. Deshalb war es notwendig, die Lage jedes einzelnen historischen Fundorts, insbesondere in der Grenzregion zu Montenegro, dem Kosovo und Mazedonien genau zu prüfen (siehe auch FrIESE, 1967).

Historische Fundorte werden mit der Schreibweise in der Originalliteratur zitiert. Unterscheiden sie sich von geläufiger und heutiger Bezeichnung, so finden sie sich im Verzeichnis in Klammern. Gehen die Fundorte auf das Zitat von FrIESE (1967) zurück, wird dies hier ebenfalls vermerkt. Stammen Nachweise des Autors ebenfalls von diesen historischen Fundorten, wird dies zusätzlich erwähnt. Alle übrigen angegebenen Fundorte gehen auf Nachweise des Verfassers zurück.
Alle Fundorte werden landesweit von Nord nach Süd durchnummeriert, wobei in der Aufstellung zu den einzelnen Provinzen (Lage und Ausdehnung erfolgt in Anlehnung an das Kartenwerk „albania, Reise Know-How Verlag Bielefeld, 2004“) chronologisch verfahren wird. In der karthographischen Darstellung wird bei den Fundorten jeweils dem jüngsten Nachweis der Vorrang gegeben.

Bei der Beschreibung der einzelnen Arten werden die Fundorte nur in der Kurzform ohne Entfernungs- und Höhenangaben genannt. Die Lage aller Fundorte ist in der Karte 1 aufgeführt.

Provinz Malësia e Madhe:

Fundort 1: Vermosh, 1.100-1.300 m NN (= Vermosha; Vermoshi) (FrIESE, 1967: 428)

Fundort 2: Han i Grabom, 205 m NN (FrIESE, 1967: 415)

Fundort 3: Umg. Bzhetë SE, Nordhänge am Kuhora e Lohes, 550 m NN (= Kunora e Lobës)

Fundort 4: Umg. Bzhetë NE, Skhel, $600 \mathrm{~m} \mathrm{NN}$

Fundort 5: Bogë, $920 \mathrm{~m} \mathrm{NN}$ und Umg. Bogë E, $1.050 \mathrm{~m} \mathrm{NN}$

Fundort 6: Umg. Bogë E, Malësia e Madhe, Südhänge am Bridashës, 1.400-1.550 m NN (Abb. 1)

Fundort 7: Umg. Bzhetë SW, 1 km SW, 490 m NN

Provinz Skodër

Fundort 8: Maja e Maranajt, 1.576 m NN (= Maranai) (FRIESE, 1967: 419, 421)

Fundort 9: Skodër (= Shkodra, Skutari) (FrIESE, 1967: 426); Skodër S, 20 m NN (WEIDLICH)

Fundort 10: Skodër-Brdica

Fundort 11: Zuri bei Skodër N, 10 m NN

Fundort 12: Umg. Paçram W bei Skodër S, 10 m NN

Fundort 13: Umg. Mjedë $3 \mathrm{~km} \mathrm{E,} 200 \mathrm{~m} \mathrm{NN}$

Fundort 14: Qafë Qelë $1 \mathrm{~km} \mathrm{E,} 500 \mathrm{~m}$ NN (= Quafa e Laçit)

Fundort 15: Vau-Deja, 80 m NN

Provinz Pukë

Fundort 16: Gjegjan, 320 m NN

Fundort 17: Umg. Gomsiqe Epërme NE, $350 \mathrm{~m} \mathrm{NN}$ (Abb. 3)

Fundort 18: Kçirë, 2 km W, 600 m NN

Fundort 19: Umg. Fushë Arrëz, 500-700 m NN

Fundort 20: Umg. Lajthizë E, 500 m NN

Provinz Kukës

Fundort 21: Beshtriku, Gebirgszug (= Bëstrique; = Bështriq) (FrIESE, 1967: 410)

Fundort 22: Kula e Lumës, 236 m NN (FrIESE, 1967: 421). Historischer, oft zitierter Fundort am Steinhaus beim Zusammenfluss des Lumës und des weißen Drins nordöstlich von Kukës. Dieser Fundort ist heute überflutet, da in den 70er Jahren des vorigen Jahrhunderts der Stausee Ligeni Fierzës angelegt wurde, der ihn und Teile der Altstadt von Kukës überflutete 
Fundort 23: Kruma, 434 m NN (FrIESE, 1967: 417)

Fundort 24: Bicaj, 413-446 m NN (Friese, 1967: 410)

Fundort 25: Mali i Gjalicës, Gebirgskette (= Djalica e Lumës) (Friese, 1967: 413, 420)

Fundort 26: Umg. Megullë, 2,5 km W, 750 m NN

Fundort 27: Umg. Kalimash, 1,5 km S, 480 m NN

Provinz Lezhë

Fundort 28: Umg. Balldren S und N, 5-20 m NN

Provinz Dibër

Fundort 29: Maja e Korabi, höchster Gipfel Albaniens, 2.764 m NN (= Korab) (Friese, 1967: 416)

Fundort 30: Kunora e Lurës, Umg. Lura (= Lurja), 1.400$2.000 \mathrm{~m}$ NN (FrIESE, 1967: 419) (Nationalpark Lura $=$ Parku Kombëtar Lura)

Provinz Kurbin

Fundort 31: Mamurras, $51 \mathrm{~m} \mathrm{NN} \mathrm{(=} \mathrm{Mamuras;} \mathrm{Mamu-}$ rasi) (FRIESE, 1967: 421)

Provinz Krujë

Fundort 32: Fuzhë Krujë, 70-80 m NN

$\underline{\text { Provinz Durrës }}$

Fundort 33: Durrës, $10 \mathrm{~m}$ NN (= Durc; Durrësi; Durazzo) (Friese, 1967: 413; WEIDLICH)

Fundort 34: Maminas, $30 \mathrm{~m} \mathrm{NN}$

Fundort 35: Rubjek, 40 m NN

Provinz Tiranë

Fundort 36: Tiranë, $121 \mathrm{~m} \mathrm{NN} \mathrm{(=} \mathrm{Tirana)} \mathrm{(FrIESE,}$ 1967: 427)

Fundort 37: Iba (=Ibë) unterhalb Krrabë (=Krraba), $400 \mathrm{~m} \mathrm{NN}$ (FrIESE, 1967: 415)

Fundort 38: Umg. Tiranë SE, $1 \mathrm{~km}$ W Mullet, $100 \mathrm{~m} \mathrm{NN}$ Fundort 39: Prezë, $90 \mathrm{~m} \mathrm{NN}$

Provinz Elbasan

Fundort 40: Umg. Elbasan E, Umg. Grigan S, 150 m NN

Fundort 41: Umg. Elbasan W, $350 \mathrm{~m} \mathrm{NN}$

Fundort 42: Umg. Elbasan, 2 km S Gracen, 650 m NN

Provinz Librazhd

Fundort 43: Babjen, 666 m NN (= Babia; Babja) (FrIESE, 1967: 410)

Fundort 44: Librazhd SE, $250 \mathrm{~m} \mathrm{NN}$

Fundort 45: Umg. Librazhd, 7 km W, 200 m NN

Provinz Lushnjë

Fundort 46: Rogozhinë, $0,5 \mathrm{~km} \mathrm{~N}$ am Skumbinid, $10 \mathrm{~m} \mathrm{NN}$

Fundort 47: Lushnjë SW, 10 m NN

Fundort 48: Kolonjë1 km S, 7 m NN

Provinz Fier

Fundort 49: Pojani, $30 \mathrm{~m} \mathrm{NN}$ (antike Stadt Apollonia) (FrIESE, 1967: 423)
Fundort 50: Fieri, 22 m NN (= Fjeri) (FrIESE, 1967: 413)

Fundort 51: Mbrostar, $8 \mathrm{~m} \mathrm{NN}$

Provinz Skrapar

Fundort 52: Poliçan im Usumi-Tal, 500 m NN (Friese, 1967: 423)

Provinz Pogradec

Fundort 53: Umg. Pogradec N, Udënisht, 700 m

Provinz Korçë

Fundort 54: Umg. Korçë S, Umg. Boboshticë, 1.100 m NN

Fundort 55: Umg. Korçë NE, M. Morave, 1 km NE Plasë, $650 \mathrm{~m} \mathrm{NN}$

Fundort 56: Umg. Korçë S, Floq 2 km W, 900 m NN

Provinz Devoll

Fundort 57: Umg. Korçë NE, M. Morave, 0,5 km SE Cangonj, $650 \mathrm{~m} \mathrm{NN}$

Fundort 58: M. Gorbeç, Umg. Bilisht E, 980 m NN

Provinz Mallakastër,

Fundort 59: Malësia i Lunxhërise, Umg. Dragot E, Vjosës - Tal, 150 m NN

Fundort 60: Dragot $1 \mathrm{~km} \mathrm{NE,} \mathrm{Vjosës} \mathrm{-} \mathrm{Tal,} 200 \mathrm{~m} \mathrm{NN}$

Fundort 61: Visokë 0,5 km NE, $100 \mathrm{~m} \mathrm{NN}$

Fundort 62: Umg. Greshicë, $300 \mathrm{~m} \mathrm{NN}$

Fundort 63: Umg. Dames, $300 \mathrm{~m} \mathrm{NN}$

Fundort 64: Umg. Memaliaj, 150 m NN

Provinz Përmet

Fundort 65: Umg. Përmet SE, W Petran, 260 m NN

Fundort 66: Umg. Kanikol E, $300 \mathrm{~m} \mathrm{NN}$

Fundort 67: Umg. Çarshovë $1 \mathrm{~km} \mathrm{W,} 350 \mathrm{~m} \mathrm{NN}$

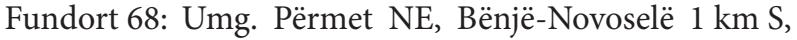
$350 \mathrm{~m} \mathrm{NN}$ (Abb. 2)

Provinz Kolonjë

Fundort 69: Umg. Leskovik W, Radova 1 km S, 600 m NN

Fundort 70: Umg. Leskovik N, Germenji $3 \mathrm{~km} \mathrm{~N}$, $1.050 \mathrm{~m} \mathrm{NN}$

Fundort 71: Umg. Leskovik N, Shales ca. 2 km SE, 900 m $\mathrm{NN}$

Fundort 72: Umg. Leskovik N, Umg. Gozharazhde E, $900 \mathrm{~m} \mathrm{NN}$

Provinz Vlorë

Fundort 73: Pasha liman, $0 \mathrm{~m}$ NN (= Paša liman) (FrIESE, 1967: 422)

Provinz Sarandë

Fundort 74: Borsh, 50-150 m NN (= Borshi) (FrIESE, 1967: 411)

Provinz Gjirokastër

Fundort 75: Umg Vrisera 0,5 km N, 300 m NN 
Fundort 76: Umg. Goranxi N, 250 m NN

Fundort 77: Umg. Gjirokastër, 18 km N, 200 m NN

\section{Systematisch-faunistisches Verzeichnis der Psychiden Albaniens}

Es werden alle bekannten Literaturauswertungen aufgeführt und das vorliegende Material detailliert beschrieben. Weitgehend verzichtet im Zitat wird jedoch auf die Literatur (z. B. Bestimmungswerke, Nachbarfaunen), die lediglich allgemein "Albanien“ nennt ohne spezifische Angaben.

Bei den Bemerkungen werden spezielle Hinweise zur jeweiligen Art bzw. zu ihren Vorkommen in den Nachbarländern Montenegro, Kosovo, Mazedonien und Griechenland gegeben. Auf die allgemeinen Verbreitungsangaben bei SAUTER \& HäTtensCHWILER (1996) und SовсZук (2011) wird nur in Einzelfällen zurückgegriffen, da nicht immer nachvollziehbar ist, woher diese stammen. Somit stellen die regionale faunistische Literatur sowie die eigenen Aufsammlungen die Grundlagen dieser Arbeit dar.

\section{Abkürzungen:}

lux = Lichtfang der Albanienexpedition des DEI 1961, i.A. = in Anzahl (3-9 Exemplare),

i.M. = in Menge (ab 10 Exemplare).

Psychidae BoISDUVAL, 1829

Naryciinae TutT, 1900

Naryciini TutT, 1900

1. Diplodoma laichartingella (GoEZE, 1783)

\section{Literatur:}

Borshi, lux 1 o 14. - 27.V.1961, leg. Friese (Petersen, 1964: 378, 379).

\section{Bemerkungen:}

Dies ist der bisher einzige Nachweis aus Albanien; auch aus Mazedonien bekannt (Thurner, 1940: 34; PeterSEN, 1964: 378; WeIDlich, 1989: 10). Neuerdings ist die Art in Griechenland nachgewiesen worden (Epirus, Voria Pindos, Umg. Aristi, 640 m NN, 1 Sack 26.04.1999, leg. Weidlich, sowie aus dem Notia Pindos, Weidlich, 2008: 499). Bei Soвсzyк (2011:111) für Griechenland nicht erwähnt.

\section{Taleporiinae HERRICH-SCHÄFFER, 1857}

Taleporiini TutT, 1900

\section{Taleporia tubulosa (RETZIUs, 1783)}

\section{Literatur:}

Kula e Lumës, 1 o 09.06.1918, leg. Penther \& Zerny (Rebel \& Zerny, 1931: 156).

\section{Untersuchtes Material:}

Balldren, mehrere Säcke 13.04.2008, leg. WEIDLICH; Bzhetë-Kurora e Lohes, ein Sack 13.04.2008; Umg. Bogë, 920-1.050 m NN, 10 Säcke 28.04.2013, leg. WEIDLICH.

\section{Bemerkungen:}

Aus allen Nachbarländern bekannt; so aus Montenegro (Rebel, 1913: 333; Rebel \& ZeRny, 1931: 156; VASIČ et al., 1990: 110, 111; WeIdlich, 2013e: in Druck), Kosovo (Rebel, 1917b: 766; Rebel \& ZeRny, 1931: 156; WeIDLICH, 2013e: in Druck), Mazedonien (DANIEL, 1965: 55; RetzlafF, 1973: 78; WeIdlich, 2013b: 27; 2013e, in Druck) und Griechenland (WeIDLICH, 2008: 499; 2013b: 27; 2013c: 962; 2013e: in Druck).

Weiterhin wurde 2013 eine cytochemische Analyse von DNA-Material vom Fundort Bogë, 1.050 m NN durchgeführt. Das Ergebnis der Analyse bestätigte die Artzugehörigkeit. Über das Portal http://www.boldsystems. org/index.php können einzelne Analysen eingesehen werden.

\section{Pseudobankesia sp.}

\section{Neu für Albanien!}

\section{Untersuchtes Material:}

Vrisera, 1 Sack 06.04.2006, aus dem am 17.04.2006 die typischen Jungräupchen schlüpften; Malësia i Lunxhërise, Dragot, 1 Sack 11.04.2008; Bënjë-Novoselë, 2 Säcke 17.04.2012, alles leg. WEIDLICH.

\section{Bemerkungen:}

Die bisherigen Sackfunde lassen bisher keine Artbestimmung zu. Ähnliche Säcke sind auch von verschiedenen Fundorten in Griechenland bekannt (WEIDLICH, 2008: 477, 499 und unveröffentlicht). Bei dem vorliegenden Material handelt sich jedoch nicht um Pseudobankesia macedoniella (REBEL, 1920), deren Säcke völlig anders gestaltet sind (WEIDLICH, 2013b: 25, 27). 


\section{Typhoniinae LEDERER, 1853}

\section{Typhoniini LEDERER, 1853}

\section{Typhonia cf. ciliaris ssp. melana (FrIVALDSZky, 1837)}

\section{Literatur:}

Korab, 2 우 23. - 31.VI.1918, leg. Predota \& Zerny; Vermosha, leg. Penther (als Melasina lugubris (HüвNer, 1808) bei Rebel \& Zerny, 1931:157; PeterSEN, 1964: 379); Petran, Säcke i.A. 11.04.2008, 1 o $^{\text {t }}$ e.l. 02.09.2008, leg. WEIDLICH.

\section{Bemerkungen:}

Bisher wurden alle Nachweise von dieser Gattung zu den Taxa ciliaris (OchSENHEIMER, 1810), melana (FRIVALDSKY, 1837), christenseni (HäTTENSCHWILER, 1990) und beatricis HätTENSCHWILER, 2000 gestellt. Die Untersuchungen zu den Typhonia-Arten des Balkans sind noch nicht abgeschlossen (WeIDLICH in Vorbereitung).

Aus den Nachbarländern gibt es Meldungen von T. ciliaris aus Montenegro (Rebel, 1913: 333; Rebel \& Zerny, 1931: 157; VAsič et al. (1990: 110), Kosovo (Rebel \& Zerny, 1931: 157), Mazedonien (DANiel, Forster \& Osthelder, 1951: 76; KAsy, 1961: 81; DANiel, 1964: 55; Weidich, 1989: 10; Huemer et al., 2011: 174) und aus Griechenland (WeIDLICH, 2008: 499).

Der Autor fand diverse cf. Typhonia-Säcke an folgenden Fundorten: Kanikol, 2 Säcke 11.04.2008; Radova, 3 Säcke 12.04.2008; Grigan, 1 Sack 13.04.2008, Bezhetë-Skhrel, 1 Sack 13.04.2008, Librazhd, SE, 1 Sack 14.04.2010, Gozharazhde, 2 Säcke 18.04.2012.

\section{Psychinae BoISDUVAL, 1840}

Psychini BoISDUVAL, 1840

\section{Luffia lapidella (GOEZE, 1783)}

\section{Neu für Albanien!}

\section{Untersuchtes Material:}

Mullet, 1 Sack 13.04.2010, leg. WEIDLICH.

\section{Bemerkungen:}

Aus den Nachbarländern war die Art bisher nur aus Griechenland bekannt. Die Funde aus dem ehemaligen Jugoslawien (SAUter \& HätTENSCHWILER, 1996: 42; SoвCZYк, 1011: 172) stammen aus Kroatien. Den Erstnachweis für Montenegro stellt der Fund eines einzelnen Sackes vom 11.04.2012, Grbalj, Umg. Kotor S, Radanovici N, $60 \mathrm{~m} \mathrm{NN}$, leg. WeIDLICH, dar.

In den letzten Jahren auch in Slowenien gefunden (WeIDLICH, 2013d: 163).

\section{Psyche crassiorella (BRUAND, 1851)}

\section{Literatur:}

Skutari/Shkodra, Säcke 05.1905, leg. Sturany (Rebel, 1913: 320; ReBeL \& ZeRnY, 1931: 124); Albanien (ReBel, 1914b: 42); Nordalbanien, mehrere Säcke 1914, leg. PENTher (Rebel, 1917a: 22; Rebel \& Zerny, 1931: 124); Kula e Lumës, 4 ơ $^{\circledR}$, 2 Säcke 07. - 14.06.1918, leg. PreDOTA \& ZERNY; Shkodra, 4 sehr große Säcke 31.07.1918, leg. Ebner (Rebel \& Zerny, 1931: 124).

\section{Untersuchtes Material:}

Borshi, i.A. Säcke, 14. - 27.05.1961, DEI; Gjirokastro, i.M. Säcke, 06.04.2006, 3 o $^{\top}$ o $^{7}, 4$ 우 ㅇ e.l. 14. - 23.05.2006; Germenji, 2 Säcke 12.04.2008; Gracen, i.A. Säcke 13.04.2008; Balldren, i.M. Säcke 13.04.2008 $70^{\pi} 0^{\top}, 13$ 우 우 e.l. 29.04. - 03.05.2008, i.A. Säcke 16.04.2012, 2 ㅇ ㅇ e.l. 03. - 05.05.2012; Bzhetë-Skhrel, i.A. Säcke 13.04.2008; Bzhetë-Kurora e Lohes, ein Sack 13.04.2008; Bogë-

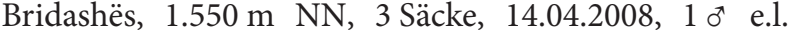
28.05.2008; Gjegjan, 4 Säcke, 13.04.2010, 1 o e.l. Anfang 05.2010; Mullet, 2 Säcke 13.04.2010; Shkodër S, i.A. Säcke 16.04.2011; Qafë Qelë, 1 Sack, 16.04.2011 und 1 Sack 28.04.2013; Gomsiqe Epërme,1 Sack 16.04.2011; Kçirë, 2 Säcke, 16.04.2011; Fushë Arrëz, 7 Säcke 17.04.2011; Fuzhë Krujë, i.A. Säcke, 16.04.2012; Maminas, i.A. Säcke 16.04.2012, $60^{\star} \sigma^{\star}$ e.l. 05. - 12.05.2012; Rubjek, i.A. Säcke, 16.04.2012, 4 o $^{\top} \sigma^{\top}, 1$ क e.l. 06.05.2012; Memaliaj, 1 Sack 17.04.2012, Bënjë-Novoselë, 2 우 우 e.l. 21.05. und 05.06.2012; Umg. Bogë, 920-1.050 m NN, 5 Säcke, 28.04.2013, 1 ơ e.l. 19.05.2013; Vau-Deja, 1 Sack 29.04.2013; Lajthizë, i.A. Säcke 29.04.2013, 1 o $^{*}$ e.l. 19.05.2013; Kalimash, i.A. Säcke 29.04.2013, alles leg. WEIDLICH.

\section{Bemerkungen:}

Eine relativ häufig vorkommende und weit verbreitete Psychidenart in Albanien, die auch aus allen Nachbarländern bekannt ist.

\section{Epichnopteriginae TuTt, 1900 \\ Epichnopterigini Tutr, 1900}

\section{Bijugis bombycella (DENIS \& SchIFFERMÜLLER, 1775)}

\section{Literatur:}

Kula e Lumës, zahlreiche, relativ kleine $0^{\top} 0^{\top}$, ein Sack 18. - 28.05, 07. - 14.06., 26.06. - 03.08.1918, leg. PreDOTA \& ZeRny (Rebel \& ZeRNY, 1931: 123).

\section{Bemerkungen:}

Die Art kommt auch in Mazedonien vor (Thurner, 1938: 179; Daniel, Forster \& Osthelder, 1951: 32; DANIEL, 1964: 53), wobei ebenfalls die geringe Größe der $0^{A} 0^{*}$ dieser Populationen hervorzuheben ist. 
SAUTER \& HÄTtenschWiler (1996: 43) sowie SobCzyK (1011: 70) geben die Art aus Griechenland an.

\section{Heliopsychidea graecella (MıLLIÈRE, 1866)}

\section{Literatur:}

Pojani, 1 o 23.06.1918, leg. Bartha (Rebel \& Zerny, 1931: 123).

Untersuchtes Material:

Vrisera, $2 \sigma^{\star} \sigma^{\star}$ und 2 weibliche Säcke 06.04.2006, 1 i e.p. 22.04.2006, Radova, 1 o 12.04.2008; alles leg. WeIDLICH.

\section{Bemerkungen:}

Diese Art kommt nur auf dem südlichen Balkan vor und ist von Millière (1866: 252) aus Griechenland beschrieben worden. Nachweise in den letzten 50 Jahren sind aus Mazedonien (DANIEL, 1964: 55, WEIDLICH, 1989: 8, 10; unveröffentlicht), Griechenland (THURNER, 1967: 19; WeIDLICH, 1989: 8, 10; 2008: 477; WEIDLICH, 2012c: 1246, 1255; 2013a: 148; 2013c: 951, 962) und aus Bulgarien (WEIDLICH, 1989: 8, 10; unveröffentlicht) bekannt geworden (vergl. auch SAUTER \& HäTteNSCHWILER, 1996: 43; SOBCZYK, 2011: 141).

\section{Montanima predotae SIEDER, 1949}

\section{Literatur:}

Djalica e Lumës, $3 \sigma^{\top} \sigma^{\top}$ 17. - 26.06.1918, leg. Predota \& Zerny; Bështriq, $2 o^{\star} o^{\star}$ 04. - 14.07.1918, leg. Predota \& Zerny (Rebel \& Zerny, 1931: 123; Sieder, 1949: 9; Meier, 1964: 59; Hauser, 2007: 144).

??? Prenj, $1.750 \mathrm{~m} \mathrm{NN}, 1$ o 18.07.1901, leg. Penther (MeIER, 1964: 59).

\section{Bemerkungen:}

Bei Rebel \& Zerny (1931: 123) und Loebel (1941: 279) noch unter karavankensis (HöfNER, 1898) geführt. Die Art ist nach $5 \sigma^{\top} \sigma^{\star}$, die auch ReBel \& Zerny (1931: 123) nennen, beschrieben worden (SIEDER, 1949: 9). MEIER (1964: 59) lagen ebenfalls $5 \sigma^{\top} \sigma^{\top}$ vor. Eigenartigerweise gibt er ein $\sigma^{\star}$ von „Prenj, $1750 \mathrm{~m}, 18.7 .1901$, leg. PeNTHER “ an. Das dritte $\sigma^{\star}$ aus der Djalica e Lumës wird nicht genannt. In Albanien gibt es eine Ortschaft Prenjs (= Prenjës) in der Provinz Elbasan (vergl. Friese, 1967: 423). Es steht jedoch ausser Zweifel, dass PeNTHER im Jahre 1901 nicht in Albanien sammelte, sondern erst später (1914) dort seine ersten entomologischen Daten erhob (vergl. Rebel, 1917a: 17; Rebel \& Zerny, 1931: 44). Der Ort Prenj liegt in den Dinariden von Bosnien-Herzegowina. Es kann sich also nur um eine Verwechslung handeln, denn selbst Meier (1964:60) schreibt über die Verbreitung: "Gebirge von Albanien". M. predotae ist bisher nur aus den Helleniden Albaniens bekannt, nicht aus dem Dinarischen Gebirge wie HAUSER (2007: 143) und SOвCZYк (2011: 195) bemerken.

\section{Rebelia cf. perlucidella ssp. macedonica PINKER, 1956}

\section{Literatur:}

Shkodra, 20. - 22.06.1918; Tirana, 21. - 25.05.1918, leg. BARTha (REBel \& Zerny, 1931: 123 als surientella).

\section{Untersuchtes Material:}

Iba, lux 1 o $^{\star 17}$. - 21.06.1961, DEI.

\section{Bemerkungen:}

REBEL \& ZERNY (1931: 123) führen für die albanische Fauna drei Rebelia-Arten an: surientella (BRUAND, 1858), sapho (Milliere, 1865) und karavankensis (HöFNER, 1898). Letztere wurde später als Montanima predotae beschrieben (siehe oben). Die Fundorte von „sapho“ bei Hodža, leg. Predota \& Zerny und Žjleb, leg. Penther (Rebel \& Zerny, 1931: 123) liegen im heutigen Kosovo. R. surientella wurde von ARNSCHEID (2012: 181) als Synonym von R. plumella (OCHSENHEIMER, 1810) erkannt. HAuser (2012: 259) führt sie hingegen als westeuropäische Subspecies von R. plumella weiter. Nach SAUter \& HätTenschwiler (1996: 43) und SовсZук (2011:281, 284) sind „surientella“ und "plumella“ von West- bis Osteuropa verbreitet, wobei Bulgarien, ehemaliges Jugoslawien, Kroatien, BosnienHerzegowina und Albanien genannt werden. Außerdem nennen Thurner, (1938: 179) Rebelia sapho sowie Pinker (1956: 204, 205), Meier (1963: 109-112), Daniel (1964: 54), Retzlaff (1973: 78) und WeidLICH (1989: 10) das Taxon Rebelia macedonica Pinker, 1956 für Mazedonien und VAsıč et al. (1990: 111) für das Durmitor-Gebiet in Montenegro. Zu diesem Taxon sollte auch der von CARNELUTti \& MiChiELI (1958: 79) als „Rebelia sappho" bezeichneter Abendflieger gerechnet werden.

HAuser (2012: 277) geht in seiner Revision der Gattung Rebelia Heylaerts, 1900 nicht auf die albanischen und montenegrinischen Funde ein. Er kommt jedoch zu dem Schluss, dass auf dem südlichen Balkan, genannt werden explizit Mazedonien und Bulgarien, nur das Taxon perlucidella ssp. macedonica vorkommt. Es erscheint daher schlüssig, dass die albanischen sowie die montenegrinischen Tiere ebenfalls hierzu gehören dürften.

\section{Psychidea nudella (OchsenHeIMer, 1810)}

\section{Literatur:}

Rebel \& Zerny (1931: 123) nennen diese Art unter „Rebelia nudella O.“ von Kruma, 1 o 29.05 .1918 , leg. PREDOTA \& Zerny 


\section{Bemerkungen:}

Meier (1966: 215, 217, 220) untersuchte dieses Ex. und stellte es zu Psychidea nudella. Die Verbreitung auf dem Balkan ist bis heute noch ungeklärt. Die Angaben für Bulgarien (REBEL \& ZERNY, 1931: 123; SAUTER \& HäTTENSCHWILER, 1996: 43; SOBCZYK, 2011: 262) können auch auf Psychidea balcanica (WEHrLI, 1933) zutreffen.

\section{Epichnopterix hellenidensis WEIDLICH, 2013}

\section{Literatur:}

Umg. Leskovik W, Umg. Radova 1 km S, 3 o o 12.04.2008; Malësia i Lunxhërise, Umg. Dragot E, Vjosës - Tal, $2 \sigma^{\top} \sigma^{\star}$ e.p. 24.04., $3 \sigma^{\top} \sigma^{\star}$ e.p. 26.04., $2 \sigma^{\top} \sigma^{\top}$ e.p. 29.04. und $2 \sigma^{\top} \sigma^{*}$ e.p. 30.04.2008, 3 ㅇ ㅇ e.p. 25.04., 1 ㅇ e.p. 30.04.2008 und 11 Säcke 11.04.2008, alles leg. WeIDLICH (WEIDLICH, 2013a: 145, 146).

\section{Untersuchtes Material:}

Radova, $3 o^{\star} o^{\star}$ 12.04.2008; Malësia i Lunxhërise, Dragot, 11 Säcke 11.04.2008, $2 \sigma^{\top} \sigma^{\top}$ e.p. 24.04., $3 \sigma^{\star} \sigma^{\star}$ e.p. 26.04., $2 \sigma^{\star} \sigma^{\star}$ e.p. 29.04. und $2 \sigma^{\star} \sigma^{\star}$ e.p. 30.04.2008, 3 i + e.p. 25.04., 1 ㅇ e.p. 30.04.2008, alles leg. WEIDLICH.

\section{Bemerkungen:}

Die Art scheint auf ihre Vorkommen in Südalbanien und dem griechischen Epirus beschränkt zu sein. Völlig unklar sind die „Epichnopteryx“ - Angaben bei Rebel \& ZERNY (1931: 123; siehe unten).

\section{Stichobasini Dalla Torre \& StRAND, 1929}

\section{Stichobasis helicinoides (HEYLAERTS, 1879)}

\section{Literatur:}

Umg. Leskovik N, Umg. Gozharazhde E, $900 \mathrm{~m} \mathrm{NN}$ 5 Säcke 12.04.2008, 2 Säcke 18.04.2012, leg. M. WEIDLICH (WEIDLICH, 2012b: 44).

\section{Untersuchtes Material:}

Gozharazhde, 5 Säcke 12.04.2008, 2 Säcke 18.04.2012, leg. WeIDLICH.

\section{Bemerkungen:}

Dieser Balkan-Endemit wurde bisher nur in Griechenland und Albanien nachgewiesen.

\section{Oiketicinae HERRICH-SCHÄFFER, 1850}

Acanthopsychini Tutr, 1900

\section{Oiketicoides lutea (STAUDIngER, 1871)}

\section{Neu für Albanien!}

Untersuchtes Material:

Megullë, 1 Sack 17.04.2011, leg. WEIDLICH.

\section{Bemerkungen:}

O. lutea ist aus Griechenland vom Veluchi-Gebirge (= Timfristos) beschrieben worden und kommt in diesem Land weit verbreitet und nicht selten vor. REBEL \& ZERNY (1931: 123) listen die Art für Albanien; die Angaben stammen jedoch aus dem Galiciča planina Mazedoniens. In Mazedonien ist O. lutea ebenfalls weit verbreitet (vergl. Drenowsky, 1930: 139, 149; Rebel \& Zerny, 1931: 123; Thurner, 1938: 178; Daniel, Forster \& Osthelder, 1951: 31; Daniel, 1964: 53; Retzlaff, 1973: 78; WeidLICH, 1989: 10).

\section{Canephora hirsuta (PodA, 1761)}

\section{Literatur:}

Nordalbanien, mehrere Säcke 1914, leg. Penther (Rebel, 1917a: 22; Rebel \& Zerny, 1931: 123); Kruma, 1 o $^{\star}$ - Sack 28.05.1918, leg. Predota \& Zerny; Mamuras, 03.08.1918, „2 Säcke fraglicher Zugehörigkeit“, leg. EbNer (Rebel \& Zerny, 1931: 123).

\section{Untersuchtes Material:}

Poliçan, 1 Sack 02. - 12.06.1961, DEI; Mullet, 2 Säcke 13.04.2010, leg. Weidlich; Udënisht, i.M. Säcke 14.04. 2010, leg. WeIdLICH; Cangonj, 3 Säcke 14.04.2010, leg. Weidlich; Umg. Bogë, 1.050 m NN, 1 Sack 28.04.2013; Bzhetë-Kurora e Lohes, 7 Säcke 28.04.2013, leg. WEIDLICH; Kalimash, 1 Sack 29.04.2013, leg. WEIDLICH.

\section{Bemerkungen:}

Auch aus allen Nachbarländern bekannt: Montenegro (Rebel, 1913: 319; Rebel \& Zerny, 1931: 123; CARneLUTTI \& MiCHIELI, 1958: 79; WEIDLICH, unveröffentlicht), Kosovo (Rebel \& ZeRnY, 1931: 123; WeIdLICH, unveröffentlicht), Mazedonien (Michieli, 1963: 25; DANIEL, 1964: 53; RetzlafF, 1973: 77; WeIDLICH, 1989: 10; WeIDLICH, M. 2012b: 27) und Griechenland (WEIDLICH, 2008: 499; WEIDLICH, 2012b: 27; 2012c: 1255, WEIDLICH, 2013c: 962).

\section{Pachythelia villosella (OCHSENHEIMER, 1910)}

Literatur:

Kula e Lumës, 4 leere Säcke und ein gezogenes $0^{*}$ 18. - 28.05., 07. - 14.06., 01.07.1918, leg. Predota \& Zerny; Paša/Pasha liman, 05.1908, leg. Winneguth (REBEL, 1913: 320; Rebel \& ZeRny, 1931: 123); Albanien (Rebel, 1914b: 42); Durc, 1 o 23.05.1917, leg. KARnY (REBel, 1918: 86; Rebel \& ZeRNY, 1931: 123). 


\section{Untersuchtes Material:}

Kunora e Lurës, 1 Sack 19. - 24.07.1961 DEI; Goranxi, i.A. Säcke 06.04.2006; Mt. Dembei, Dragot, 2 Säcke 11.04.2008; Petran, 3 Säcke 11.04.2008; Radova, 8 Säcke 12.04.2008 und 18.04.2012; Shales, 2 Säcke 12.04.2008; Floq, 2 Säcke 12.04.2008; Gracen, i.A. Säcke 13.04.2008; Balldren, i.M. Säcke 13.04.2008 und 16.04.2012; BezhetëSkhrel, 3 Säcke 13.04.2008; Mjedë, i.M. Säcke 13.04.2010, 2 우 우 e.l. 12. - 27.05.2010, $10^{\star}$ e.l. 14.05.2010; Librazhd, 7 km W, 4 Säcke 14.04.2010; Librazhd, SE 5 Säcke 14.04.2010, 1 o e.l. 28.05.2010; Shkodër S, 30 Säcke 16.04.2011; Qafë Qelë, 1 Sack 16.04.2011; Kçirë, 1 Sack 16.04.2011; Fushë Arrëz, 1 Sack 17.04.2011; Paçram, i.M. Säcke 16.04.2012, i.A. o o e.l. 05. - 17.05.2012, 1 क e.l. Anfang 05.2012; Prezë, 1 Sack 16.04.2012; Maminas, i.A. Säcke 16.04.2012; Rubjek, i.M. Säcke 16.04.2012; Durrës, i.A. Säcke 17.04.2012; Rogozhinë, i.M. Säcke, 17.04.2012; Lushnjë, 2 Säcke 17.04.2012; Kolonjë, 1 Sack 17.04.2012; Mbrostar, 2 Säcke 17.04.2012; Visokë, 20 Säcke 17.04.2012, i.A. ơ o e.l. 02. - 14.05.2012; Greshicë, i.A. Säcke 17.04.2012; Dames, i.A. Säcke 17.04.2012; Bzhetë SW, ein Sack, 28.04.2013, 1 ơ e.l. 25.05.2013; Lajthizë, 1 Sack 29.04.2013, alles leg. WeIDLICH.

\section{Bemerkungen:}

P. villosella zählt $\mathrm{zu}$ den verbreitetsten und häufigen Arten in Albanien. Ebenso aus allen Nachbarländern bekannt und in etlichen Literaturstellen zitiert, worauf an dieser Stelle verzichtet werden kann.

\section{Oreopsychini TUTT, 1900}

\section{Ptilocephala plumifera (OCHSENHEIMER, 1810)}

\section{Literatur:}

Bështriq, 1 ơ 29.04. - 04.06.1918, leg. Predota \& Zerny (REBEL \& ZERNY, 1931: 123).

\section{Untersuchtes Material:}

Bogë-Bridashës, $1.400 \mathrm{~m} \mathrm{NN}, 4 o^{\star} o^{\star} \quad 14.04 .2008$, leg. WEIDLICH.

\section{Bemerkungen:}

Rebel \& Zerny (1931: 123) stellen das vorliegende o zur ssp. mediterranea (LEDERER, 1853). Nach heutiger Kenntnis ist diese Unterart auf die Alpen der Schweiz, Österreichs und Italiens beschränkt. P. plumifera ist ebenfalls aus den Nachbarländern Mazedonien (DANIEL, Forster \& OSTHELDER, 1951: 31; DANIEL, 1964: 53) und Montenegro (VAsiČ et al. 1990: 112; WEIDLICH, unveröffentlich), weiterhin aus Griechenland und Bulgarien (WeIdLICH, 1989: 9, 10; WeIdlich, 2008: 499; WeIdLICH, 2013c: 951, 962) bekannt. Die Nachweise für Griechenland fehlen bei SoвCZYк (2011: 275).

\section{Phalacropterigini TutT, 1900}

\section{Megalophanes viciella (Denis \& SchifFERMüLlER, 1775)}

Literatur:

Bështriq, 1 leerer Sack, leg. Predota \& Zerny (Rebel \& ZERNY, 1931: 123).

\section{Bemerkungen:}

Die Art ist hier in ihrer ssp. orientalis DIERL, 1974 vertreten und auch aus den Nachbarländern Montenegro (VAsič et al., 1990: 112, 113), Kosovo (Rebel \& ZeRnY, 1931: 123) und Mazedonien (REBEL, 1913: 320; REBEL \& Zerny, 1931: 123; Daniel, Forster \& Osthelder, 1951: 31; DANIEL, 1964: 53, 54; WeIDLICH, 1989: 10) publiziert. SAUTER \& HÄTTENSCHWILER (1996: 45) geben die Art für Griechenland an.

\section{Loebelia crassicornis (StAUDINGER, 1871)}

\section{Literatur:}

Durc, 1 o 24.04.1917, leg. Karny (Rebel, 1918: 86; Rebel \& Zerny, 1931: 123; Rebel, 1940: 61; WeIdLiCH, 2012: 80); Vrisera 2 Säcke, 06.04.2006; Radova, 5 Säcke 12.04.2008 und 5 Säcke 17.04.2012; Mullet, 2 Säcke 13.04.2010, alles leg. WeIDLICH (WEIDLICH, 2012a: 80).

\section{Untersuchtes Material:}

Vrisera, 2 Säcke 06.04.2006; Radova, 5 Säcke 12.04.2008 und 5 Säcke 18.04.2012; Mullet, 2 Säcke 13.04.2010; Visokë, 10 Säcke 17.04.2012, alles leg. WEIDLICH.

\section{Bemerkungen:}

Die Verbreitung des Balkan-Endemiten, welcher auch in den östlich angrenzenden Ländern Mazedonien und Griechenland vorkommt, hat WeIDLICH (2012a) zusammenfassend dargestellt. Demnach erreicht die Art bei Dürres und Tiranë ihre Arealnordwestgrenze.

Apteronini TUTT, 1900

\section{Apterona helicoidella (VALLOT, 1827)}

\section{Literatur:}

Skutari-Brdica, Säcke 1905, leg. Sturany (Rebel, 1913: 320); Albanien (Rebel, 1914b: 42); Bështriq, Säcke, leg. Predota \& Zerny; Shkodra, leg. Sturany; Han i Grabom, leg. Penther (Rebel \& Zerny, 1931: 123).

\section{Untersuchtes Material:}

Mt. Dembei, Dragot, 1 Sack 11.04.2008; Germenji, i.A. Säcke 12.04.2008; Gozharazhde, 1 Sack 12.04.2008; Boboshticë, 1 Sack 12.04.2008; Grigan, i.A. Säcke 13.04. 
2008; Elbasan, 2 Säcke 13.04.2008; Bzhetë-Skhrel, 2 Säcke 13.04.2008; Mjedë, i.M. Säcke 13.04.2010; Librazhd SE, i.A. Säcke 14.04.2010; Udënisht, i.M. Säcke 14.04.2010; Plasë, i.M. Säcke, 14.04.2010; Cangonj, hunderte Säcke 14.04.2010; Bilisht, i.M. Säcke 14.04.2010; Qafë Qelë, i.A. Säcke 16.04.2011; Fushë Arrëz, i.A. Säcke 16. - 17.04.2011; Visokë, i.A. Säcke 17.04.2012; Bogë, 920 m NN, 2 Säcke, 28.04.2013; Lajthizë, 3 Säcke 29.04.2013, alles leg. WEIDLICH.

\section{Bemerkungen:}

Die relativ häufige und verbreitete Art ist ebenfalls aus allen Nachbarländern bekannt.

\section{Incertae sedis}

\section{Eumasia parietariella (HEYDENREICH, 1851)}

\section{Literatur:}

Bicaj, 14. - 15.06.1918; Kula e Lumës, mehrfach; $40^{\top} o^{\star}$ 26.VI. - 03.VII.1918, leg. Predota \& Zerny (Rebei \& Zerny, 1931: 158; Petersen, 1964: 378), Borshi, lux 6 o o 14. - 27.V.1961, leg. Friese (Petersen, 1964: 378).

\section{Untersuchtes Material:}

6 o o Borshi, lux 14. - 27.V.1961, leg. FrIESE); Gjirokastro, 8 Säcke, 06.04.2006, leg. WeIDLICH; Malësia i Lunxhërise, Dragot, i.A. Säcke, 11.04.2008, leg. WeIDLICH; Bezhetë-Skhrel, 2 Säcke 13.04.2008, leg. WeIdLICH; Zuri, 9 Säcke, 13.04.2010, leg. WeIDlich; Gjegjan, 1 Sack, 13.04.2010, leg. Weidlich; Bënjë-Novoselë, i.A. Säcke 17.04.2012, 1 ㅇ e.l. 02.06.2012 und $20^{\star} \sigma^{\star}$ e.l. Anfang 06.2012; Vau-Deja, 4 Säcke 29.04.2013, leg. WEIDLICH.

\section{Bemerkungen:}

Eine doch relativ wenig beobachtete Art, die lokal nicht selten auftritt. Aus fast allen Nachbarländern nachgewiesen: Montenegro (Weidlich, unveröffentlicht), Mazedonien (Thurner, 1940: 34; KASY, 1961: 81; KLIMESCH, 1968: 186; WeIDLICH, 1989: 10; unveröffentlicht) und Griechenland (Thurner, 1940: 34; KLIMEsch, 1968: 186; WeIDLICH, 1989: 10; 2008: 499; 2012c: 1255; 2013c: 962).

Die von Rebel \& Zerny, (1931: 123, 124) für Albanien genannten Proutia betulina (Zeller, 1839) aus Peć (Kosovo), Psyche casta (Pallas, 1767) und Rebelia sapho (Milliere, 1865) beide aus dem Galiciča planina (Mazedonien, $P$. casta auch bei Drenowsky, 1930: 147 aufgeführt) sowie Sterrhopterix fusca (HAwORTH, 1809) von Nove selo (Kosovo), stammen somit nicht vom albanischen Staatsterritorium. Die entsprechenden Angaben bei Sauter \& Hättenschwiler (1996) und Sobczyk (2011) sind deshalb irreführend.
Nachfolgend werden einige Ausführungen zu weiteren in der Literatur genannten

Psychidentaxa gegeben.

\section{„Solenobia inconspicuella Stt."}

Die Bezeichnung "Solenobia inconspicuella“ wurde bis weit in das 20. Jahrhundert für damals weitgehend unbestimmbare Dahlica- und teilweise auch SiederiaArten verwendet. Dahlica inconspicuella (STAInton, 1849) ist heute jedoch ausschließlich aus Großbritanien bekannt. So fand das o vom 08.05.1905 vom Maranai, leg. Sturany unter diesem Namen Eingang in die Literatur (Rebel, 1913: 333; 1914b: 43). Später ist dieses Exemplar dann unter "Solenobia Nickerlii Hein.“ geführt worden (REBEL \& ZERNY, 1931: 156). Ob es sich hier tatsächlich um eine Dahlica - Art handelt, ist unklar. Jedenfalls liegen aus Albanien keine gesicherten Nachweise irgendeiner Dahlicini-Art vor. Huemer et al. (2011: 174) nennen eine „Dahlica sp.“ aus Mazedonien.

\section{„Solenobia Nickerlii Hein."}

Vom Bëstrique ein Sack mit weiblicher Puppenhülle, 04. - 14.07.1918, leg. Predota \& Zerny, genannt (ReBeL \& ZERnY, 1931: 156). Zweifelsfrei handelt es sich nicht um Dahlica nickerlii (HeInEmanN, 1870), die ausschließlich in Mitteleuropa vorkommt.

\section{„Bankesia spec."}

In der Urbeschreibung von Pseudobankesia macedoniella, als Bankesia beschrieben, erwähnt ReBeL (1920: (144)) aus Albanien ein „fast zweifelslos zu der vorbeschriebenen B. macedoniella gehöriges, geflogenes Stück ( $o^{\star}$ ) ... am Korab in ca. 1800 m Höhe Ende Juli“. Später veröffentlichen REBEL \& ZERNY (1931:156) nochmals die bekannten macedoniella-Funde vom locus typicus = Galičica planina in Mazedonien. Das abgeflogene $\sigma^{*}$ aus dem Korab wird nicht mehr als „Bankesia macedoniella“ sondern als Bankesia spec. geführt und die Autoren geben nun an, das es „nach seinem stark geflogenem Zustand keine sichere Bestimmung, zuläßt. Nichtsdestotrotz fand dieser Fund bis heute immer wieder Eingang in die Literatur (vergl. SAUter \& HäTtenSCHWILER, 1996: 41; KARSHOLT \& NieuKERKEN, 2012; SoвCzyк 2011:248). Pseudobankesia macedoniella ist nach heutiger Kenntnis nur aus Mazedonien und Griechenland belegt (vergl. WeIdLICH, 2013b: 23-27). 
Taleporia politella (OCHSENHEIMER, 1816)

\section{Literatur:}

Kula e Lumës, mehrfach leere Säcke, leg. Penther \& ZERNY (REBEL \& ZeRnY, 1931: 156).

\section{Bemerkungen:}

Im Einzelnen gibt es für „politella“ folgende Angaben: Rebel (1914a: 1127) nennt Ricavac südwestlich von Vermosh (1300-1900 m NN) als Fundort und bezeichnet ihn zu Albanien gehörig (Rebel, 1914a: 1112). Später definieren Rebel \& Zerny (1931:156) diesen Fundort als montenegrinisch und auch FrIESE schließt sich dieser Ansicht an (Friese, 1967:431 - als Rikavac). Der Fundort Novo selo am Žljeb (vergl. ReBeL, 1917b: 766) befindet sich im Kosovo und ReBEL \& ZeRNy (1931: 156) verzeichnen Kula e Lumës als Fundort.

Die bisher publizierten Angaben von dieser Art, die ausschließlich auf Sackfunde basieren, gehören T. tubulosa an (vergl. Weiduich, 2013e: in Druck). T. politella ist hauptsächlich pannonisch verbreitet und die nächsten gesicherten, aktuellen Vorkommen liegen bei Senj in Kroatien.

\section{Eochorica balcanica (ReBel in Prinz, 1919)}

\section{Literatur:}

Albanien (Sauter \& HätTenschwiler, 1996: 42; SobCZYK, 2011: 116).

\section{Bemerkungen:}

Bereits Petersen (1964: 379) vermutet das Vorkommen dieser Art in Albanien. Belegmaterial aus diesem Land konnte der Autor jedoch nicht ermitteln. Für die Nachbarländer liegen Angaben aus Mazedonien (Rebel in PrINZ, 1919: (134); 1940: 62-64; Drenowsky, 1930: 137; ThurNER, 1936: 15; 1940: 34; DANIEL, 1964: 55; RetZlafF 1973: 79; WitT, 1985: 48, 49; Weidlich, 1989: 5, 10; unveröffentlicht; SAUTER \& HäTtENSCHWILER, 1996: 42) und Griechenland (WItT, 1985: 48; WeIDlich, 1989: 10; 2013c: 951, 962; SAUTER \& HÄTtensCHWILER, 1996: 42) vor.

Die bei cf. Typhonia angeführten Säcke aus Kanikol, Radova, Grigan, Bezhetë-Skhrel, Librazhd und Gozharazhde, können auch dieser Art angehören.

\section{"Epichnopteryx pulla (Esp.) Sieboldi Reutti"}

Rebel \& Zerny (1931: 123) nennen dieses Taxon (Taxone) vom Bështriq (1 ơ 29.04. - 04.06.1918, leg. Predota \& Zerny), Korab (29.07.1918 ein leerer Sack, leg. Predota \& Zerny) und Fjeri (1 o 24.06.1918, leg. BARTHA). Die systematische Stellung ist völlig unklar (siehe auch bei E. hellenidensis).
Acanthopsyche zelleri (MANn, 1855)

\section{Literatur:}

Babia, leg. Karny (Rebel, 1918: 86; Rebel \& Zerny, 1931: 123).

\section{Bemerkungen:}

Rebel (1918: 86) selbst zweifelt an der Determination und schreibt hierzu: „Babia (700 Meter) 30. Oktober (1917). Ein wahrscheinlich hierher gehöriger Sack“" Wenig später vermutet auch THURner (1938: 178), dass einige Sackfunde von der Petrina planina Mazedoniens zu A. zelleri gehören könnten. In der Mazedonienfauna von DANiel (1964) wird diese Art dann aber nicht mehr genannt.

Vor fast 150 Jahren hat ManN die Art bei Split (= Spalato) nachgewiesen (MANN, 1869:376) und Dalmatien wird auch bei Dalle Torre \& Strand (1929: 160) genannt. Die nächsten aktuellen Nachweise befinden sich im rumänischen Banat (KöNIG \& WEIDLICH, 2001: 735; RÁkosy, Goia \& Kovács, 2003: 31; Weidlich, 2013: unveröffentlicht - etwa $470 \mathrm{~km}$ entfernt) sowie auf der kroatischen Inseln Cres und Rab (WEIDLICH, unveröffentlicht - etwa $620 \mathrm{~km}$ entfernt). Nach heutiger Kenntnis kommt die Art auf dem südlichen Balkan nicht vor.

\section{Danksagung}

Meinem Freund W. ARnscheid (Wetter/Deutschland) sei an dieser Stelle für seine Unterstützung bei den Diskussionen und Korrekturen aufrichtig gedankt.

Ebenfalls gilt den Mitarbeitern des Senckenberg Deutschen Entomologischen Institutes, ehemals Deutsches Entomologisches Institut in Eberswalde am heutigen Standort Müncheberg mein herzlicher Dank.

\section{Literatur}

Arnscheid, W. R. 2012: Die Identität von Rebelia surientella (BRUAND, 1858) (Lepidoptera: Psychidae). - Entomofauna 33 (14): 181-188.

Carnelutti, J. \& Michieli, Š. 1958: I. Beitrag zur Lepidopterenfauna der Crna Gora. - Fragmenta Balcanica 2 (10): 67-81.

Dalle Torre, K. W. v. \& Strand, E. 1929: Lepidopterorum Catalogus. Pars 34. Psychidae: 211 S., Berlin.

DANIEL, F. 1964: Die Lepidopterenfauna jugoslavisch Mazedoniens. - II. Bombyces et Sphinges. - Prirodonaučen Muzej Skopje, Posebno izdanie Nr. 2: 1-74.

Daniel, F.; Forster, W. \& Osthelder, L. 1951: Beiträge zur Lepidopterenfauna Mazedoniens. - Veröffentlichungen der Zoologischen Staatssammlung München 2: 1-78, Taf. 1-2. 
Drenowsky, A. K. 1930: Beitrag zur Lepidopterenfauna S. W. Mazedoniens. - Spisanie na Bulgarskaja Akademija na Naukite 42: 129-177.

Friese, G. 1967: Ergebnisse der Albanien-Expedition 1961 des Deutschen Entomologischen Institutes. 61. Beitrag Verzeichnis albanischer Fundorte. - Beiträge zur Entomologie 17 (3/4): 405-434.

Friese, G. \& Königsmann, E. 1962: Ergebnisse der Albanien-Expedition 1961 des Deutschen Entomologischen Institutes. 1. Beitrag: Bericht über den Verlauf der Reise. - Beiträge zur Entomologie 12 (7/8): 765-843.

Hauser, E. 2007: Artspezifische Beschuppung in der Gattung Montanima SiEder 1849 und Untersuchung am Typenmaterial von Rebelia catandella Solyanikov 1998. - Linzer biologische Beiträge 39 (1): 143-153.

Hauser, E. 2012: Revision der Gattung Rebelia Heylaerts 1900 (Lepidoptera, Psychidae). - Linzer biologische Beiträge 44 (1): 181-306.

Huemer, P.; Krpač, V.; Plössl, B. \& Tarmann, G. 2011: Contribution to the fauna of Lepidoptera of the Mavrovo National Park (Republic of Macedonia). Acta Entomologica Slovenica 19 (2): 169-186.

Karsholt, O. \& Nieukerken, E. J. van 2012: Lepidoptera. Moth. Fauna Europaea, Version 2.5., http// www.faunaeur.org/distribution.php.

Kasy, F. 1961: Beiträge zur Kenntnis der Micro- und Macroheteroceren - Fauna Westmazedoniens. Zeitschrift der Arbeitsgemeinschaft österreichischer Entomologen 13 (3): 65-82.

Klimesch, J. 1968: Die Lepidopterenfauna Mazedoniens. IV. Microlepidoptera. - Prirodonaučen Muzej Skopje, Posebno izdanie Nr. 5: 1-201.

Loebel, F. 1941: Die Rebelien Mitteleuropas. - Zeitschrift des Wiener Entomologen-Vereins 26: 271-282, Taf. XXX.

MANN, J. 1869: Lepidoptern gesammelt während dreier Reisen nach Dalmatien in den Jahren 1850, 1862 und 1868. - Verhandlungen der zoologisch-botanischen Gesellschaft in Wien 19: 371-388.

Meier, H. G. 1963: Rebelia (nec Psychidea) perlucidella BRUAND (Lepidoptera: Psychidae) (4. Beitrag zur Kenntnis der Psychiden). - Zeitschrift der Wiener Entomologischen Gesellschaft 48: 105-113.

Meier, H. G. 1964: Zur Kenntnis der Gattungen Peleponnesia Sieder und Montanima Sieder (Lep., Psychidae) (6. Beitrag zur Kenntnis der Psychiden). Zeitschrift der Wiener Entomologischen Gesellschaft 49: 47-61.

Meier, H. G. 1966: Revision der Gattung Psychidea Rambur (nec Leucanacanthia Wehrli) (Lep., Psychidae) (7. Beitrag zur Kenntnis der Psychiden). - Deutsche entomologische Zeitschrift N.F. 13 (I/III): 203-230.

Michieli, S. 1963: Beitrag zur Kenntnis der Makrolepidopterenfauna Mazedoniens (S. R. Makedonija). - Acta Musei Macedonici Scientiarum Naturalium 9(2): 15-33.
Petersen, G. 1964: Ergebnisse der Albanien-Expedition 1961 des Deutschen Entomologischen Institutes. 29. Beitrag Lepidoptera: Psychidae I (Micropsychina sensu Dalla Torre \& STrand, 1929). - Beiträge zur Entomologie 14 (3/4): 377-380.

Pinker, R. 1956: Erkenntnisse, gewonnen bei Beobachtungen mazedonischer Psychiden. - Fragmenta Balcanica 1 (25): 201-205.

RÁkosy, L.; GoiA, M. \& KovÁcs, Z. 2003: Verzeichnis der Schmetterlinge Rumäniens: 446 S., Cluj-Napoca.

Rebel, H. 1913: Studien über die Lepidopterenfauna der Balkanländer. III. Teil. Sammelergebnisse aus Montenegro, Albanien, Mazedonien und Thrazien. - Annalen des k. k. naturhistorischen Hofmuseums Wien 27: 281-334.

ReBEL, H. 1914 a: Lepidopteren aus dem nordalbanischmontenegrinischen Grenzgebiete. (Ergebnisse einer von der Kaiserl. Akademie der Wissenschaften in Wien veranlaßten naturwissenschaftlichen Forschungsreise in Nordalbanien). - Sitzungsberichte der kaiserlichen Akademie der Wissenschaften Wien, mathematisch-naturwissenschaftliche Klasse, 123 (1): 1111-1128.

Rebel, H. 1914b: Zur Lepidopterenfauna Albaniens. - XVIII. und XIX. Jahresbericht des Naturwissenschaftlichen Orientvereins über die Jahre 1912 und 1913, Wien: 37-43.

Rebel, H. 1917a: Neue Lepidopterenfunde in Nordalbanien, Mazedonien und Serbien. - XXI. und XXII. Jahresbericht des Naturwissenschaftlichen Orientvereins über die Jahre 1915 und 1916, Wien: 17-24.

Rebel, H. 1917b: Lepidopteren aus Neumontenegro (Ergebnisse der im Jahre 1916 im Auftrage und auf Kosten der Kaiserl. Akademie der Wissenschaften in Wien von Dr. Arnold Penther ausgeführten zoologischen Forschungsreise in Serbien und Neumontenegro). - Sitzungsberichte der kaiserlichen Akademie der Wissenschaften Wien, mathematischnaturwissenschaftliche Klasse, $126(1)$ : 765-813, Taf. 1-5.

Rebel, H. 1918: Lepidopteren aus Mittelalbanien. Zeitschrift des Österreichischen Entomologen Vereines 3 (8): 75-77, (9): 85-88.

Rebel, H. in Prinz, J. 1919: Bericht der Sektion für Lepidopterologie. Versammlung am 7. März 1919. Beitrag zur Kenntnis paläarktischer Mikrolepidopteren. - Verhandlungen der zoologisch-botanischen Gesellschaft Wien 69: (126)-(135).

Rebel, H. 1920: Bericht der Sektion für Lepidopterologie: Bankesia macedoniella. - Verhandlungen der zoologisch-botanischen Gesellschaft Wien 69 (1919): (142)-(144).

Rebel, H. 1940: Zur Kenntnis einiger Subfamilien der Psychiden. - Zeitschrift des Wiener EntomologenVereines 25 (3): 59-65, (4): (73-76), Taf. XI.

Rebel, H. \& Zerny, H. 1931: Die Lepidopterenfauna Albaniens (mit Berücksichtigung der Nachbargebiete). - Denkschriften der Akademie der Wissenschaften in 
Wien, mathematisch-naturwissenschaftliche Klasse 103: 37-161.

Retzlaff, H. 1973: Ein Beitrag zur Psychiden-Fauna Jugoslawisch- Mazedoniens (Lep.). - Entomologische Zeitschrift 83 (6): 77-80.

Sauter, W. \& Hättenschwiler, P. 1996: Psychidae. - In: Karsholt, O. \& Razowski, J. (eds.). The Lepidoptera of Europe. A Distributional Checklist. Psychidae: 39-46, Apollo Books, Stenstrup.

Sieder, L. 1949: Montanima gen. nov. (Lep. Psychidae). Zeitschrift der Wiener Entomologischen Gesellschaft 34: 2-9, 1 Taf.

SoвсZYк, T. 2011: World Catalogue of Insects. Volume 10. Psychidae (Lepidoptera): 467 S., Apollo Books, Stenstrup.

THURNER, J. 1936: Einige bemerkenswerte Lepidopterenfunde aus Mazedonien. - Zeitschrift des Österreichischen Entomologen Vereines 21: 6-7, 13-15.

Thurner, J. 1938: Die Schmetterlinge der Ochrid-Gegend in Macedonien. - Mitteilungen der königlichen naturwissenschaftlichen Institute in Sofia, Bulgarien 11: 121-179.

Thurner, J. 1940: Die Schmetterlinge der OchridGegend in Macedonien. II. Teil: Microlepidoptera. Mitteilungen der königlichen naturwissenschaftlichen Institute in Sofia, Bulgarien 14 (1941): 9-35.

THURner, J. 1967: Lepidopteren aus Morea. Ein weiterer Beitrag zur Fauna des Peloponnes (Griechenland). Zeitschrift der Wiener Entomologischen Gesellschaft 52: 5-23, 50-58.

Vasić, K.; Tomić, D.; Carnelutti, J.; Zečević, M. \& KranjČEv, R. 1990: Heterocera I. Bombyces et Sphinges (Insecta, Lepidoptera). - Fauna Durmitora 14 (3): 99-157.

Weidlich, M. 1989: Abriß der Psychidenfauna Bulgarisch-Mazedoniens mit der Erstbeschreibung des Weibchens und des Sackes von Reisseronia nigrociliella (Rebel, 1934) (Lepidoptera, Psychidae). - Nachrichtenblatt der Bayerischen Entomologen 38 (1): 1-12.

Weidlich, M. 2008: Beitrag zur Lepidopteren-Fauna des Notia Pindos (Tringia-Massiv, Lákmos-Gebirge und Athamáno-Gebirge) in Griechenland mit Beschreibung von zwei neuen Arten sowie Angaben zur Köcherfliegen- (Trichoptera) und Schnakenfauna (Diptera: Tipulidae). - Entomofauna 29 (27): 469-504.

Weidlich, M. 2012a: Zur Kenntnis von Loebelia crassicornis (Staudinger, 1871) (Lepidoptera: Psychidae). - Entomologische Zeitschrift 122 (2): 79-85.

WeIDLICH, M. 2012b: Zur Verbreitung und zur Biologie des Balkanendemiten Stichobasis helicinoides (HEYLAERTs, 1879) (Lepidoptera: Psychidae). - Microlepidoptera.hu 5: 43-48.

WeIDlich, M. 2012c: Zur Schmetterlings-Fauna der Zagoria im Voria Pindos (Griechenland) mit der Beschreibung einer neuen Psychidenart (Lepidoptera, Psychidae). - Linzer biologische Beiträge $44(2)$ : 1237-1262.
Weidlich, M. 2013a: Eine neue Psychide aus den Helleniden Griechenlands und Albaniens (Lepidoptera: Psychidae). - Nachrichten des entomologischen Vereins Apollo N.F. 33 (4): 145-148.

WeidLICH, M. 2013b: Zur Verbreitung des Balkanendemiten Pseudobankesia macedoniella (REBEL, 1920) (Lepidoptera: Psychidae). - Microlepidoptera. hu 6: 23-27.

WeIDLICH, M. 2013c: Zur Schmetterlings-Fauna des Pangéo-Gebirges in Griechisch-Mazedonien mit Beschreibung von Dahlica pangeoensis nov. sp. und Bemerkungen zur Köcherfliegenfauna (Lepidoptera, Trichoptera). - Linzer biologische Beiträge 45 (1): 945-969.

Weidlich, M. 2013d: The bisexual Dahlica triquetrella (HÜBNER, 1813) and new records of Luffia lapidella (GoEze, 1783) in Slovenia (Lepidoptera: Psychidae). - Acta Entomologica Slovenica 21 (2): 163-168.

WeIdlich, M. 2013e: Über die südliche Arealgrenze von Taleporia tubulosa (Retzius, 1783) auf dem Balkan sowie Bemerkungen zum Areal von Taleporia politella (Ochsenheimer, 1816) (Lepidoptera, Psychidae). Entomologische Zeitschrift: in Druck.

WitT, T. J. 1985: Neue und bemerkenswerte Heterocerennachweise aus Griechenland (Lepidoptera, Arctiidae, Lymantriidae, Thyatiridae, Psychidae). - Nachrichtenblatt der Bayerischen Entomologen 34 (2): 47-49. 


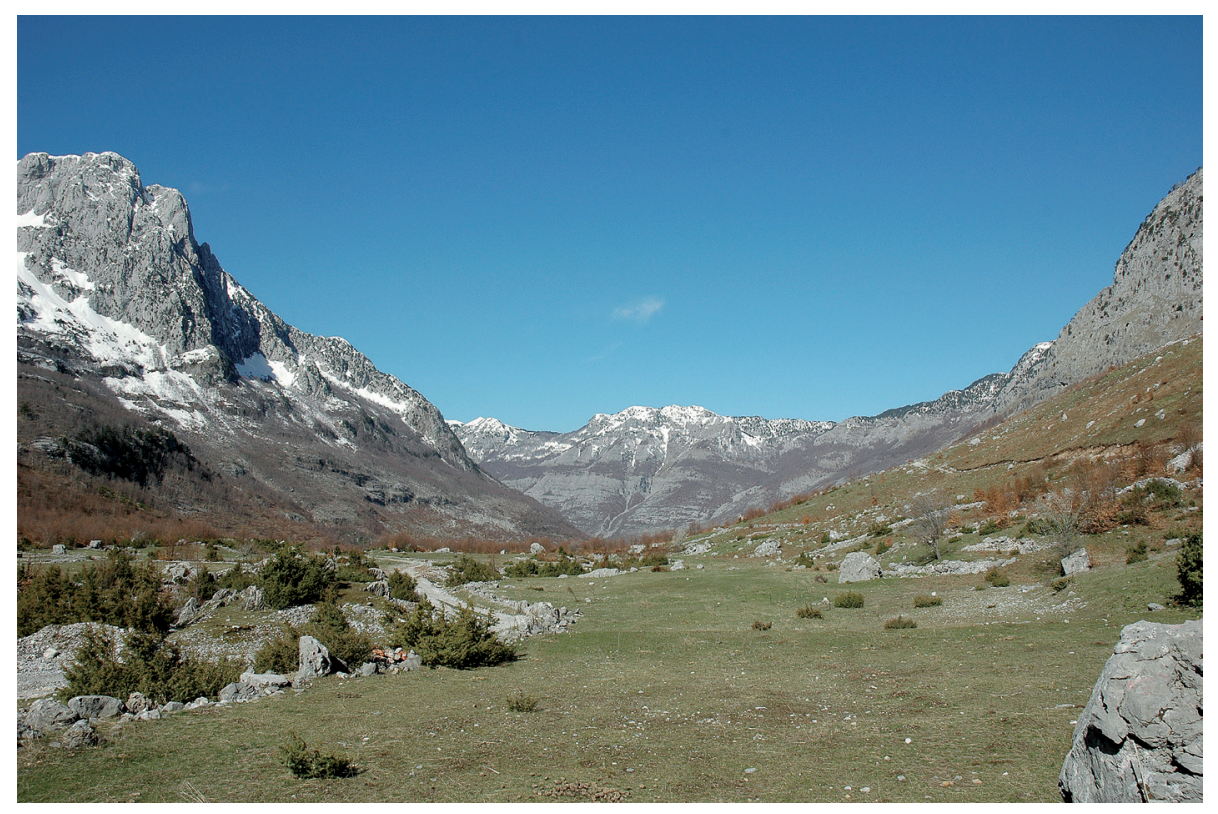

Fig. 1: In den nordalbanischen Alpen, Provinz Malësia e Madhe, Umg. Bogë E, Südhänge am Bridashës, 1.400$1.550 \mathrm{~m} \mathrm{NN}$, Lebensraum von Ptilocephala plumifera $\mathrm{O}$. und Psyche crassiorella BRD. (Foto: 14.04.2008).

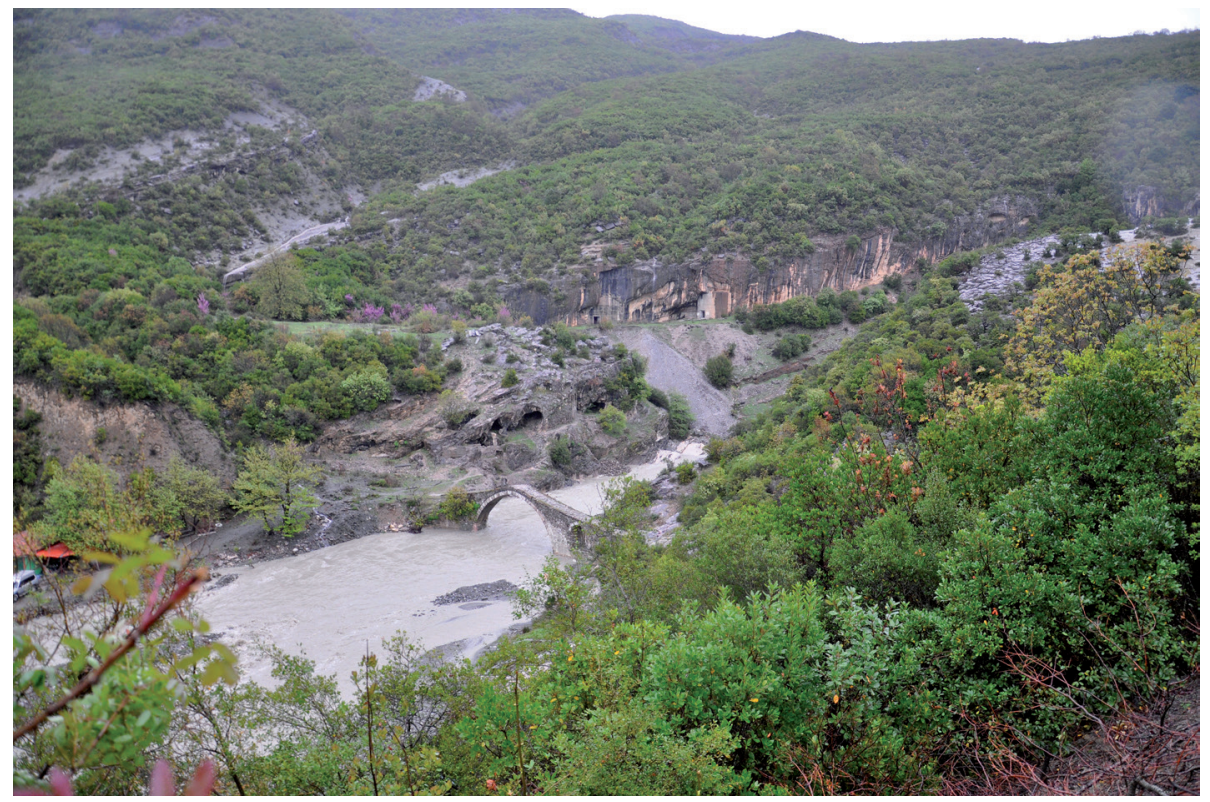

Fig. 2: In Südostalbanien, Provinz Përmet, Umg. Bënjë-Novoselë $S$, $350 \mathrm{~m} \mathrm{NN}$, Lebensraum von Pseudobankesia sp., Psyche crassiorella BRD. und Eumasia parietariella Heyd. (Foto: 17.04.2012).

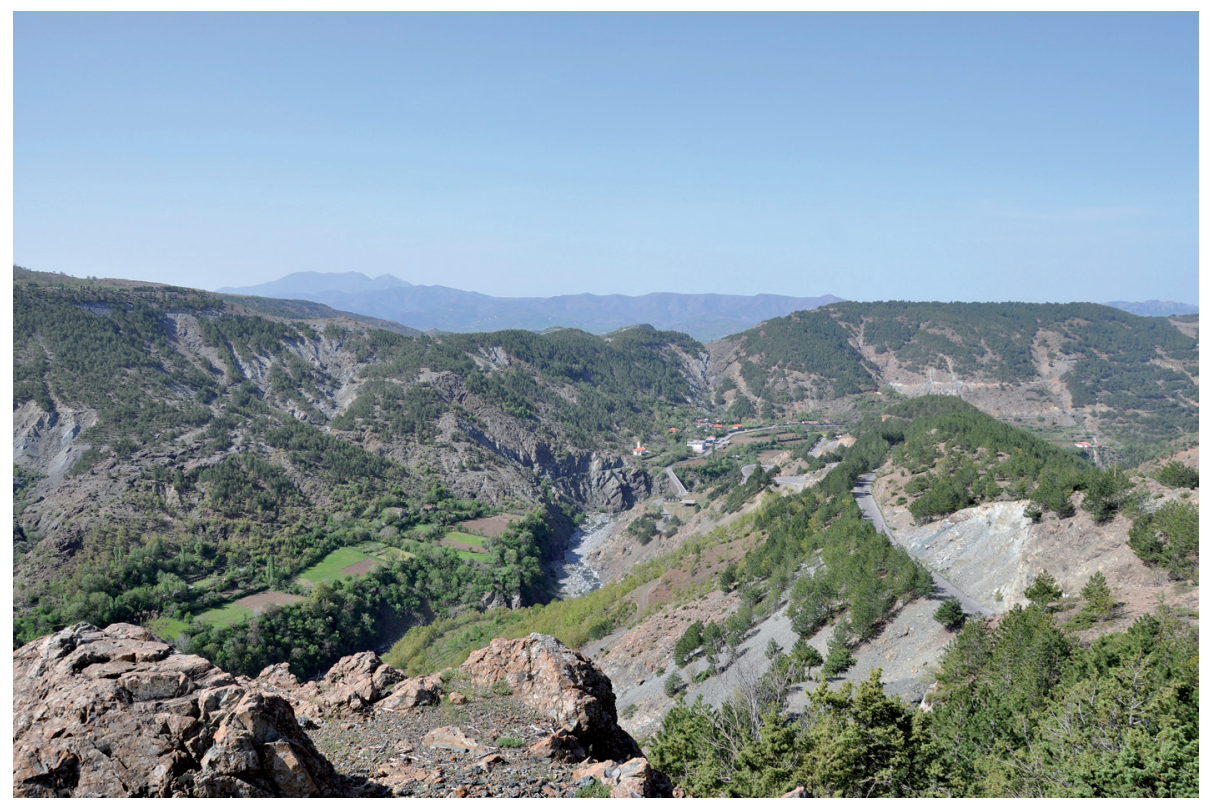

Fig. 3: In Nordalbanien, Provinz Pukë, Umg. Gomsiqe Epërme NE, $350 \mathrm{~m}$ NN, Lebensraum von Psyche crassiorella BRD. (Foto: 29.04.2013).

Alle Fotos: Dr. M. WeIDLICH 